\title{
FUNGAL DYSBIOSIS CORRELATES WITH THE DEVELOPMENT OF TUMOUR-INDUCED CACHEXIA IN MICE
}

\author{
Jabes, D.L. ${ }^{1}$, de Maria, Y.N.L.F. ${ }^{1}$, Aciole Barbosa, D. ${ }^{1}$, Santos, K.B.N.H. ${ }^{1}$, Carvalho, L.M. ${ }^{1}$, \\ Humberto, A.C. ${ }^{1}$, Alencar, V.C. ${ }^{1,2}$, Costa de Oliveira, R. ${ }^{1}$, Batista Jr, M.L. ${ }^{1}$, Menegidio, F.B. ${ }^{1}$, Nunes, \\ L.R. ${ }^{2 *}$
}

1.Núcleo Integrado de Biotecnologia, Universidade de Mogi das Cruzes (UMC), Brazil, 2.Centro de Ciências Naturais e Humanas, Universidade Federal do ABC (UFABC), Brazil

*To whom correspondence should be addressed: Luiz R. Nunes, PhD, Centro de Ciências Naturais e Humanas,

Universidade Federal do ABC, Alameda da Universidade, S/N, São Bernardo do Campo, SP, 09606-045, Brazil. Tel:

(55)(11) 4996-8371/4996-3166; E-mail: Luiz.nunes@ufabc.edu.br

\begin{abstract}
Cachexia (CC) is a devastating metabolic syndrome associated with a series of underlying diseases that greatly affects life quality and expectancy among cancer patients. Studies involving mouse models, in which CC was induced through inoculation with tumor cells, originally suggested the existence of a direct correlation between the development of this syndrome and changes in the relative proportions of several bacterial groups present in the digestive tract. However, these analyses have focus solely on the characterization of bacterial dysbiosis, ignoring the possible existence of changes in the relative populations of fungi, during the development of CC. Thus, the present study sought to expand such analyses, by characterizing changes that occur in the gut fungal population (mycobiota) of mice, during the development of cancer-induced cachexia. Our results confirm that cachectic animals display significant differences in their gut mycobiota, when compared to healthy controls. Moreover, identification of dysbiotic fungi showed remarkable consistency across successive levels of taxonomic hierarchy. Many of these fungi have also been associated with dysbioses observed in a series of gut inflammatory diseases, such as obesity, Colorectal Cancer (CRC), Myalgic Encephalomyelitis (ME) and Inflammatory Bowel Disease (IBD). Nonetheless, the CC-associated dysbiosis seems to be unique, presenting features observed in both obesity (reduced proportion of Mucoromycota) and CRC/ME/IBD (increased proportions of Sordariomycetes, Saccharomycetaceae and Malassezia). One species of Mucoromycota (Rhyzopus oryzae) stands out as a promising probiotic candidate in adjuvant therapies, aimed at treating and/or preventing the development of CC.
\end{abstract}

Keywords: Microbiome; Microbiota; Mycobiota; Cachexia; Next Generation Sequencing; $N G S$. 


\subsection{INTRODUCTION}

Cachexia (CC) is recognized as a metabolic syndrome associated with several underlying diseases, such as cancer, chronic kidney disease and chronic heart disease, among others $(1,2)$. It is characterized by the reduction of muscle mass, depletion of body fat and generalized chronic inflammation (3). Its prevalence among patients with different types of cancer contributes to significantly decrease their life quality and expectancy, being an important cause of morbidity/mortality in more than $80 \%$ of advanced cancer cases and accounting for more than $20 \%$ of deaths $(3,4,5)$. Until the 1980 s, cachexia was attributed to anorexia and increased energy expenditure. However, enteral or para-enteral administration of nutritional supplements is not sufficient to reverse cachexia symptoms, refuting the hypothesis that nutrient deficiency is the main causative agent of this syndrome. During the late 1980s and early 1990s, cachexia came to be defined through a new concept, as a chronic inflammatory syndrome, and the metabolic changes associated with the cachectic condition derive from factors produced by both external agents (such as tumors), as well as by the affected organism (2).

Studies aimed at characterizing the microbiota of mice, during the development of cancer-induced cachexia, revealed significant alterations in the composition of their gut bacterial population (dysbiosis), when compared to healthy control animals $(6,7,8,9,10,11)$. The main characteristic of such dysbiosis involves an increased proportion of Enterobacteriaceae in $\mathrm{CC}$-affected animals - a phenomenon also observed during the development of several diseases and syndromes associated with gut inflammation, such as celiac disease (12), food allergy (13), gastric cancer (14), diabetes $(15,16)$, obesity $(17,18,19)$ and inflammatory bowel diseases (IBD), such as ulcerative colitis (UC) and Crohn's disease (CD) (20), among others. More recently, cultivation of fecal material from cachectic mice in selective coliform media, identified Klebsiella oxytoca as the main representative of Enterobacteriaceae present in stool samples obtained from these animals (21). Based on this observation, it has been suggested that $K$. oxytoca could represent an intestinal pathobiont, capable of affecting both thickness and permeability of the mucus barrier that protects the digestive epithelium of mice, when over-represented in their gastrointestinal track $(11,21,22)$.

These findings demonstrated that gut dysbiosis could be an important factor for the development of cancer-induced cachexia, raising the interesting possibility of developing adjuvant approaches to prevent/treat cachexia, by controlling the gut microbiota composition with the aid of specific antibiotics, prebiotics and probiotics. However, all CC studies carried out so far have focused solely on analyzing the bacterial microbiota, ignoring the possible existence of changes in the relative populations of fungi during the development of this syndrome. Thus, the present study sought to expand the scope of such analyses by characterizing the main alterations verified in the fungal population (mycobiota) present in the guts of mice, during the development of cancer-induced cachexia. In general, it was possible to verify the existence of significant differences involving the gut mycobiota of CC animals, in comparison to healthy controls. The fungal dysbiosis of the cachectic animals revealed alterations in the relative proportions of several fungal taxa, consistently distributed in successive levels of taxonomic hierarchy. In many cases, relative changes in these populations have also been described in dysbioses associated with other gut inflammatory diseases, although the $\mathrm{CC}$-associated dysbiosis seems to display a unique signature. Moreover, some of the microorganisms found to be expanded among $\mathrm{CC}$-affected animals display metabolic characteristics that may directly contribute to the development of cachexia. Finally, we identified fungal species preferably expanded in the gut of healthy 
animals, which can be grown axenically, under laboratory conditions. These latter microorganisms represent natural candidates to be tested as probiotics in adjuvant therapies, aiming at the treatment and/or prevention of cachexia.

\subsection{MATERIALS AND METHODS}

\subsection{CACHEXIA INDUCTION IN C57BL/6 MICE AND COLLECTION OF STOOL SAMPLES FOR GUT MYCOBIOTA CHARACTERIZATION}

The experiments described herein were conducted with 6-8-week old male mice, weighting 25-38 g. Animals were selected from colonies of C57BL/6 mice (Jackson Laboratory), which were bred and kept in plastic cages, in a rodent-only controlled environment, with low levels of external noise, $24 \pm 1{ }^{\circ} \mathrm{C}$ average temperature and light/dark cycles every 12 hours. Food (Nuvilab CR1 ${ }^{\circledR}$ - Nuvital) and water were provided ad libitum. All animals were individually marked with identifying rings at the beginning of the experiment. A group of 10 animals was randomly chosen as a control group (CT), having received only subcutaneous injection with $200 \mu \mathrm{l}$ saline solution in their right flank. The others (20) were similarly inoculated with $3.5 \times 10^{5}$ LLC tumor cells (suspended in $200 \mu \mathrm{l}$ of Dulbecco's modified Eagle's medium). Next, the animals were transferred to sterile cages and kept, under the conditions described above, for a period of 28 days, when cachexia symptoms could be verified $(23,24)$. Stool samples were collected daily, from each mouse, until day 28. All samples were properly identified, frozen in liquid $\mathrm{N}_{2}$ and stored at $-70^{\circ} \mathrm{C}$. The genetic material obtained from the samples collected at day 28 was used to characterize the fecal microbiota of both CT and LLC-inoculated animals, as described below. All animals were sacrificed by decapitation, without anesthesia, immediately after collecting the last stool samples. Blood, organ and tissue samples from these mice were harvested and frozen at $-70^{\circ} \mathrm{C}$. This experimental design was evaluated and approved by the Ethics Committee for the Use of Animals in Research, at the University of Mogi das Cruzes (CEUA-UMC), under records n: 006/2013 and n: 009/2013.

\subsection{ASSESSMENT OF CACHEXIA PROGRESSION}

After inoculum, either with saline solution or LLC cells, all animals were weighed daily, on a precision scale (always at the same time), throughout the entire course of the experiment. This information was used to calculate the Cachexia Index (CI) in the LLCinoculated animals, following the methodology described in 24. According to these calculations, 8 animals showed CI values $\geq 5$, indicating full development of cachexia (24). These animals were further evaluated for their cachectic condition by histometric characterization of muscle (gastrocnemius) and adipose (epididymal) tissues (25), as well as by measuring the expression of molecular markers for muscular atrophy (Atrogin) and inflammation (IL6 receptor), by real-time quantitative PCR (qPCR) (see 26,27,28), for details). These 8 animals were designated Cachectic Group, or CC, which was further used for subsequent microbiome analyses. A similar group of 8 animals (the Saline Control Group, or SC) was selected among the CT animals, to establish a control group of similar size for future comparisons with the $\mathrm{CC}$ group.

\subsection{SAMPLE PROCESSING AND DNA EXTRACTION}


The extraction of fungal DNA from stool samples was performed with the aid of the DNeasy Power Soil kit, according to the manufacturer's instructions (Qiagen). Integrity of the extracted material was evaluated through electrophoresis in a $1.5 \%$ agarose gel and purity was estimated through their $\mathrm{A}_{260} / \mathrm{A}_{280}$ ratios, using a NanoDrop 2000 spectrophotometer (Thermo Scientific). The final quantification of each DNA sample was determined with the aid of a Quantus fluorometer, according to the manufacturer's specifications (Promega). The material from these extractions was used to characterize the fungal mycobiota by NGS sequencing, as described below.

\subsection{PREPARATION AND SEQUENCING OF ITS1 AMPLICON LIBRARIES}

Fungal populations were evaluated by comparing sequences from ribosomal internal spacer region 1 (ITS1), as described by 29. All primers used for library construction had, at their 5 'ends, complementary regions to Illumina's Nextera XT adapters. Thus, the amplicons obtained from each sample were subjected to a second round of PCR with Nextera adapters, adding specific barcode sequences at their ends. The entire process for preparing and purifying the amplicon libraries was performed as recommended by Illumina (https://support.illumina.com/documents/documentation/chemistry_documentation/16s/16smetagenomic-library-prep-guide-15044223-b.pdf). Average sizes of the generated fragments were evaluated after electrophoresis in a Bioanalyzer 2100 (Agilent), using the High Sensitivity DNA Chip and the libraries were quantified by qPCR, in an ABI Prism 7500 Fast Sequence Detection System (Applied Biosystems), with the aid of the universal quantification kit NEBNext (New England Biolabs), according to the manufacturer's instructions. The final products of amplification were then aliquoted in a mixed sample of equimolar concentration and subjected to paired-end sequencing ( 2 X 300) in an Illumina MiSeq DNA sequencer, according to the manufacturer's specifications (Illumina, Inc). Raw sequences obtained during the development of this study have been deposited at the Open Science Framework (https://osf.io/5fxgn) and SRA repository (https://www.ncbi.nlm.nih.gov/sra), under Provisional Submission ID \# SUB7683387.

\subsection{AMPLICON SEQUENCE ANALYSIS AND MYCOBIOTA CHARACTERIZATION}

Sequence quality of individual reads was initially evaluated with the aid of FastQC, v 0.11. 9 (https://www.bioinformatics.babraham.ac.uk/projects/fastqc/) and MultiQC, v 1.7 (30), and subsequently analyzed through the QIIME toolkit, v 1.9.1 (31). During the preprocessing stage, individual reads were demultiplexed, paired and integrated into full size amplicons. Next, a filtering step removed all poor-quality amplicons from further analysis (filtered elements included amplicons that displayed overlapping reads with less than 30 bases, strings with more than $10 \%$ divergence in the overlapping region, or that had more than three bases with quality index below Q20). Next, FASTA files from the selected amplicons were submitted to USEARCH v. 8 (32) to identify and eliminate chimeric artifacts, using the UNITE database, v. 8.0, as a reference $(33,34)$.

During the processing stage, Operational Taxonomic Units (OTUs) were created, by clustering the individual sequences with a $97 \%$ identity threshold, with the aid of SortMeRNA (35) and SumaClust (36) software, using the Open Reference method. Taxonomic annotation was performed using the RDP classifier algorithm (37), with default 
settings, and the UNITE database v. 8 as reference (34). The resulting OTU Table was then filtered to remove contaminants (non-fungal, bacterial, plastid and archaea data) and singletons. Finally, a minimal abundance filter $(\mathrm{A}<0.00001)$ was applied to remove OTUs underrepresented in the microorganism population. All filtering steps were performed using QIIME commands, as described by 38 .

All these analytical steps have been implemented in a workflow described in a Jupyter Notebook file (39), in a Docker container, based on the Dugong project (40) and made available through BioPortainer Workbench (41). Details regarding this workflow can be found at: https://osf.io/5fxgn (DOI 10.17605/OSF.IO/5FXGN).

\subsection{FUNGAL POPULATION AND DIVERSITY ANALYSES OF THE GUT MYCOBIOTA FROM SC AND CC MICE}

Measurement of population ratios and Alpha/Beta diversity analyses were conducted with the aid of MicrobiomeAnalyst $(42,43)$, using the OTU Table described above. To load the data, the Low Count Filter was adjusted to minimum values (Minimum Count $=0$ and Prevalence in Sample $=10$ ) and the OTU Table was rarefied by the number of sequences in the smallest library (Rarefy to the Minimum Library Size). All other parameters remained unchanged. Statistical significance of differences involving alpha diversity measurements between SC and CC groups was evaluated by a T-test (using $\mathrm{p} \leq 0.05$ as threshold). Variations in beta diversity were conducted with the aid of Bray-Curtis metrics (44) and visualized by a Non-linear Multidimensional Scaling (NMDS) algorithm, followed by statistical validation with PERMANOVA (using $\mathrm{p} \leq 0.05$ as threshold) (45). All these analyses were conducted using tools directly provided by MicrobiomeAnalyst. Dysbiosis Ratios (DR) were calculated and statistically validated as described in 46, using population ratios obtained from MycrobiomeAnalyst.

\subsection{IDENTIFICATION OF DIFFERENTIALLY REPRESENTED TAXA IN SC AND CC MICE, USING LINEAR DISCRIMINATIVE ANALYSYS EFFECT SIZE (LEfSe).}

Initially, core microbial populations were determined for each group separately (SC and CC), using the QIIME command compute_core_microbiome.py to select only OTUs present in $\geq 70 \%$ of the animals in the group. The OTU Tables containing the SC and CC Core 70 microbiotas were then integrated, using the QIIME merge_otu_tables.py command, resulting in a Core 70 OTU Table for the entire SC X CC dataset. The use of $70 \%$ as a threshold to define Core microbiotas followed other studies described in the literature, where such limits vary between $50 \%$ and $80 \%(47,48,49,50,51,52,53,54,55,56)$. Next, the Core 70 OTU Table was submitted to the QIIME summarize_taxa.py script, integrating the results obtained for each taxon and converting their absolute prevalences into relative ratios. This relative OTU Table was then submitted to a Linear Discriminant Analysis Effect Size (LEfSe) evaluation, using the default settings of the Galaxy LEfSe tool, available at https://huttenhower.sph.harvard.edu/galaxy/. 


\subsection{RESULTS}

\subsection{CACHEXIA INDUCTION AND CHARACTERIZATION IN MICE}

A group of 20 mice was submitted to LLC tumor cell transplantation and monitored for a 28-day period, under controlled conditions, as described in Materials and Methods. At the end of this period, animals were sacrificed and their weight gain was compared to a group of 10 control animals (CT group), to determine their respective Cachexia Indexes (CI) (Figure 1). Animals displaying CI $\geq 5$ were further evaluated for other cachexia criteria, which included the observation of morphometric changes in adipose and muscle tissues (average decreases in adipocyte size and in the area occupied by muscle fibers, with consequent increase of interstitial muscular tissue), as well as increased expression of marker genes for muscular atrophy and inflammation (Atrogin and IL6R, respectively) $(25,26,27,28)$ (Figure 1). In total, 8 LLC-injected animals displayed all characteristics of cachexia described above and these mice were selected for microbiome analyses, constituting the $\mathrm{CC}$ group. Accordingly, we selected a group of equal size among the CT animals to constitute the Saline Control (SC) group, which were used for further comparisons with the CC animals. Thus, stool samples obtained from SC and CC mice, at day 28, were further processed to characterize their fungal gut populations (mycobiota).

\subsection{CHARACTERIZATION AND COMPARISON OF FUNGAL GUT POPULATIONS (MYCOBIOTA) IN SC AND CC ANIMALS}

Amplicon libraries referring to the ITS1 sequences were built for the animals of both SC and CC groups, as described in Materials and Methods and subjected to sequencing in an Illumina MiSeq DNA sequencer. The number of amplicons obtained for each animal, after all pre-processing steps, varied between 66,436 and 163,377, as shown in Supplementary Table 1 . These sequences were submitted to an analytical pipeline, specially developed in our laboratory, generating a table of Operational Taxonomic Units (OTU Table) that was later used to analyze and compare the fungal gut populations (mycobiota) of SC and CC animals.

Surprisingly, initial analyses revealed that there was no alteration in fungal alphadiversity when the fecal mycobiota of SC and CC animals were compared, since the absolute number of fungal species and OTUs did not show statistically significant differences (Figure 2A). Similar results were observed when using alternative alpha-diversity metrics (Shannon, Chao1, ACE, Simpson and Fisher) and/or after extending the analyses to other taxonomy levels (phylum to genus) (not shown). However, a beta diversity analysis revealed that the SC and CC animals display sufficient differences in the composition of their gut mycobiota to discriminate the two groups (Figure 2B). This analysis was performed with the aid of a Non-linear Multidimensional Scaling (NMDS) algorithm, using the Bray-Curtis index to compare the SC and CC mycobiota contents. The result from this analysis was subsequently evaluated by PERMANOVA (45), 2001), confirming that the SC and CC groups exhibit sufficient differences in the composition of their gut fungal populations to allow their discrimination at statistically significant levels $(\mathrm{p}=0.045)$, although the two main components of the NMDS analysis do not provide visual separation between the two groups (Figure 2B). 
As observed in Figure 3A, the vast majority of the fungal population in the gut of both SC and CC animals involves members from the phyla Ascomycota ( 85-87\%), Basidiomycota ( $\sim 12-13 \%)$ and Mucoromycota ( 0.4-1.5\%). When the two groups are compared, however, it is possible to verify that $\mathrm{CC}$ animals display statistically significant reduction $(\sim 16 \mathrm{X})$ in both MucoromycotalAscomycota and Mucoromycota/Basidiomycota ratios (Figure 3B-C). It is also possible to observe a slight increase $(\sim 1.7 \mathrm{X})$ in the Ascomycota/Basidiomycota ratio of CC animals, although this difference did not display statistical significance under our experimental conditions (Figure 3D). Interestingly, similar population imbalances involving the main fungal phyla have also been observed in association with alternative gut inflammatory diseases and syndromes (see below). Unfortunately, it was not possible to detect statistically significant alterations involving other groups of fungi below the level of phylum, probably due to limitations involving the use of classical univariate statistical inference methods on microbiota compositional data (see 57).

To overcome such limitations, the mycobiota of SC and CC animals were further compared through LEfSe (Linear discriminant analysis Effect Size), a widely used tool specifically developed to identify differentially represented elements from microbiome data (58) (Figure 4). This analysis was conducted with a Core 70 OTU Table, containing only OTUs present in, at least ,70\% of the animals in each group. This strategy is often used to reduce effects related to individual variability among subjects, minimizing the effect of taxa that are over-represented in few individuals within a group, but not consistently present throughout the group $(47,48,49,50,51,52,53,54,55,56)$. As expected, LEfSe analysis confirmed that members of Mucoromycota were present at higher proportion in the stool samples of SC animals, when compared to CC. Moreover, the SC-overrepresented Mucoromycota identified by LEfSe displayed remarkable consistency across all hierarchical levels of fungal taxonomy, involving representatives from the Mucoromycetes class, Mucorales order and Rhizopodaceae family, with emphasis on the species Rhizopus arrhizus, also classified as $R$. oryzae (59).

LEfSe also confirmed that there was no significant alteration in the overall distribution of Ascomycota and Basidiomycota phyla between SC and CC animals, but detected a series of fungal groups belonging to these two phyla that were differentially distributed in the stool samples from both SC and CC groups (Figure 4). Interestingly, the fungal groups that displayed the most consistent distribution across successive hierarchical levels of taxonomy highlight a series of taxa (mostly Ascomycota) that seem to be preferably expanded among CC animals (Figure 4). These include, for example, representatives from all taxonomic levels of the Sordariomycetes class, including members of the Hypocreales order and Nectiriaceae family, with emphasis on a species of Fusarium, with $\underline{u}$ nspecified classification [wuc]. Members of the Saccharomycetaceae family also showed population expansion in the stool samples of cachectic animals, including representatives of Saccharomyces and Kazachstania (with emphasis on S. cerevisiae and K. pintolopesii, respectively). Two representatives of Tallaromyces ( $T$. stollii and another species [wuc]) were also identified as preferentially present in the feces of cachectic mice, when compared with SC animals. Additional Ascomycota displaying population expansion among CC animals involve representatives of the Pleosporales order, including members [wuc] of the Didymellaceae family. Interestingly, only two species of Basidiomycota were identified as over-represented in the feces of CC animals and both belong to the genus Malassezia, which congregates fungi traditionally involved with inflammatory processes, especially in the skin (M. japonica and $M$. dermatis). Animals from the SC group, on the other hand, presented expanded populations of Basidiomycota from the Cystobasidiomycetes class, including 
members of the Cystobasidiales order and Cystobasidiomycetaceae family, with emphasis on the species Cystobasidium slooffiae. Only three species of Ascomycota were identified as expanded among SC animals: Cladosporium haloterians, Penicillium citrinum and one species [wuc] of Aspergillus.

Finally, to test the reliability of these results, the relative proportions of some of the taxa described above were evaluated in SC and CC animals, using real-time quantitative PCR (qPCR). For this purpose, equimolar samples of DNA extracted from the stool samples from each mouse (the same DNAs used for the construction of amplicon libraries) were consolidated into two pools, representing the SC and CC groups. Samples of these pools were then used in qPCR experiments, with the aid of primers previously described in the literature as specific to the genera Saccharomyces, Kazachstania and Malassezia, as well as to the species Saccharomyces cereviseae, Malassezia dermatis, Malassezia japonica, Rhizopus oryzeae and Penicillium citrinum $(60,61,62,63,64,65,66,67)$. The $C t$ values obtained for each of these taxa were normalized by the $C t$ obtained with qPCR amplifications using the primer pairs used to build the fungal amplicon libraries and the relative quantification values, obtained for each taxon, are shown in Figure 5. These experiments confirmed the results obtained by LEfSe analysis, showing a significant expansion of representatives of Saccharomyces and Kazachstania (as well as the species $S$. cereviseae) within the gut of animals from the CC group, with more than one order of magnitude, in relation to SC. Representatives of the genus Malassezia (including $M$. dermatis and $M$. japonica) were also more represented in the pool of cachectic animals, although in smaller proportions ( 3 to $5 \mathrm{X}$ ). Representatives of Rhizopus oryzeae, on the other hand, were detected exclusively in the pool of SC animals, which also corroborates the results obtained from LEfSe analysis. The only taxon that could not be amplified in these qPCR experiments was Penicillium citrinum, but we do not know if such negative results derive from under-representation of these fungi in the mycobiota from the two groups, or from problems regarding efficiency of the primers used in these experiments.

\subsection{DISCUSSION}

Cachexia represents one of the most devastating clinical conditions that can occur with individuals affected by different types of cancer, since its high degree of morbidity results in significant reduction in the patients' life quality. Moreover, treatments aimed at preventing or combating the development of CC often meet with limited success, since its onset and progression may be influenced by a variety of internal and external factors (2). In this sense, the existence of correlations between the development of cachexia and intestinal dysbiosis, as observed in mice, suggests that manipulation of gut microbial communities may assist in the development of novel adjuvant approaches to alleviate symptoms in cachectic patients, or to prevent its onset in individuals at risk of developing such syndrome. In this sense, the results presented herein demonstrate, for the first time, that intestinal dysbiosis associated with cachexia is not limited to changes in bacterial population, but also involves significant changes in the fungal population present in the gut of cachectic animals.

Analysis of relative ratios involving the main phyla of fungi are commonly performed to establish fungal dysbiosis ratios (DR), which have been proposed as quantitative indexes to describe alterations in mycobiota that accompany the progression of other dysbiosis-associated diseases $(46,68)$. In this sense, the fungal DR observed in the gut of CC animals displays significant alterations in the relative ratios of both MucoromycotalAscomycota and Mucoromycota/Basidiomycota populations, confirming that 
CC animals display reduced proportions of Mucoromycota in their gut. Interestingly, a similar scenario has been reported by 69 , who compared the gut mycobiota of lean and obese individuals. This similarity is noteworthy, since obesity and cachexia are both metabolic syndromes, directly associated with imbalances in energy metabolism, which affect both fat storages and muscular tissue. In the study conducted by 69 , the relative reduction in the population of Mucor spp constituted the hallmark of the fungal dysbiosis verified in obese individuals. Thus, these authors suggested that the presence of chitosan in the cell wall of Mucor species could contribute to prevent obesity (a well-documented nutritional property of this polysaccharide) $(70,71)$.

In this sense, Rhyzopus oryzae, the main Mucoromycota involved in the CCassociated dysbiosis described herein, may also act as a potential chitosan source in the gut of healthy animals, since this species has been employed in many industrial protocols aimed at chitosan production $(72,73,74)$. This information raises the interesting possibility of testing chitosan and some of its derivatives, as food supplements, in adjuvant protocols aimed at treating or preventing cachexia, which may enhance the current pharmaceutical and biomedical applications of this polysaccharide (75). Additionally, $R$. oryzae has been reported to produce substantial amounts of various antioxidants and organic acids, including gallic acid (76). Interestingly, an extract of oil palm phenolics (OPP), containing 1,500 ppm gallic acid equivalent, has been shown to inhibit tumorigenesis, by mediating G1/S phase cell cycle arrest, delay inflammatory responses and attenuate cachexia symptoms in tumorbearing mice $(77,78)$. Gallic acid has also been shown to improve glucose tolerance and triglyceride concentration in obese mice (79) and suppress lipogenesis in humans, while concomitantly combating pro-inflammatory responses (80).

Alterations in relative ratios involving fungal phyla have also been detected and used to calculate DRs for a series of dysbiosis-associated diseases and syndromes, including Colorectal Cancer (CRC), Myalgic Encephalomyelitis (ME) (also known as Chronic Fatigue Syndrome, or CFS) and the two main forms of Inflammatory Bowel Disease (IBD): Crohn's disease (CD) and Ulcerative Colitis (UC) $(46,81,82,83,84)$. While the abovementioned diseases display alternative clinical manifestations, they all share common traits with obesity and cachexia, including increased permeability of the protective mucosal gut barrier and intestinal inflammation. Contrary to obesity and cachexia, however, CRC/ME/IDB/CD/UC do not affect adipose and muscular tissues and do not display gut dysbioses based on reduced ratios of Mucoromycota. Instead, these pathologies are all associated with fungal gut dysbioses characterized by altered Ascomycota/Basidiomycota ratios, when affected subjects are compared to their respective controls. As mentioned above, our analysis also indicated a small increase in the overall Ascomycota/Basidiomycota ratio, when CC animals are compared to SC, although this difference was not statistically significant, when evaluated by a classic Mann-Whitney U test. However, a more sophisticated comparison, performed with LEfSe, on the core mycobiotas of SC and CC animals, revealed that different groups of Ascomycota were preferentially expanded in the gut of cachectic mice an information that was further confirmed by qPCR experiments, involving a series of representative taxa.

Interestingly, the main groups of Ascomycota, whose populations are expanded in $\mathrm{CC}$ animals, exhibit similar behavior in some of the diseases mentioned above. For example, a recent study aimed at evaluating the composition of the human intestinal mycobiota, demonstrated that members of the Sordariomycetes class (especially Fusarium and Trichoderma) are significantly enriched in rectal tissue samples from patients affected by CRC (85). Several species of Fusarium are recognized for causing infections involving both 
normal and immunosuppressed individuals and toxins produced by this group of fungi have already been shown to induce cell proliferation and affect different intestinal defense mechanisms (81). For example, Fusarium toxins can severely reduce thickness of the intestinal mucus layer, increasing its permeability and allowing microorganisms present in the gut lumen to reach the intestinal wall, triggering the production of immunoglobulins and pro-inflammatory cytokines (86). In fact, at least one Fusarium toxin, known as deoxynivalenol (DON) has already been correlated with the development of IBD (87), which is also in accord with the observations made by 82 , who detected expansion of Sordariomycetes in the inflamed intestinal mucosa of CD patients.

Members of the Saccharomycetaceae family also showed increased population in the gut of patients affected by $\mathrm{CD}$, with emphasis on Candida glabrata, a pathogen known to mediate inflammatory responses in the human intestine (82). In this sense, our studies point to a significant expansion involving the populations of two species of Saccharomycetaceae, among the CC animals: Saccharomyces cereviseae and Kazachstania pintolopesii. Moreover, the population expansion involving these two yeasts seems to occur at very high rates in the gut of $\mathrm{CC}$ animals $(\sim 30 \mathrm{X})$, as verified by qPCR experiments. Interestingly, members of the Kazachstania genus show great phylogenetic proximity to Candida (88) and previous studies have even suggested that these two yeast genera could perform analogous functions in the intestinal micro-ecosystem of different animals (89). Paradoxically, studies by 82 identified an increased proportion of Saccharomyces cerevisiae in non-inflamed mucous membranes of healthy individuals, which led these authors to suggest that this yeast could play a beneficial role, preventing the development of $\mathrm{CD}$. This proposal is in accord with results derived from other studies, involving both humans and mice, which suggested that $S$. cerevisiae could exert anti-inflammatory effects in host tissues, inducing the production of interleukin-10 $(68,90)$.

However, 91 have argued otherwise, since the presence of $S$. cerevisiae exacerbated intestinal inflammation in a mice model of $\mathrm{UC}$ and increased the permeability of the protective intestinal mucosal barrier in these animals. In an independent study, 92 demonstrated that members of the Saccharomycetaceae family (particularly S. cereviseae and Kazachstania unispora) are able to trigger inflammatory responses through the interaction of $\alpha$-mannans, present in their cell walls, with the lectin dectin- 2 of their host's dendritic cells. The presence of $S$. cerevisiae also seems to potentiate the production of metabolites capable of exacerbating inflammatory processes in their hosts, including increased production and accumulation of uric acid (91). Finally, anti- S. cerevisiae antibodies (ASCAs) are often found in patients with CD and UC, being used as serological markers for differential diagnosis between these two forms of IBD $(93,94)$. Taken together, these data reinforce the existence of a positive correlation between increased intestinal populations of $S$. cereviseae and $K$. pintolopesii with the development of gut inflammatory responses and increased permeability of the protective mucosal barrier, along the intestine (two characteristics also observed during the development of cachexia).

Other examples of Ascomycota displaying population expansion in the stool samples of $\mathrm{CC}$ animals have few references (or none) of association with the development of diseases. These fungi include members of the Tricomaceae family, with emphasis on the species Talaromyces stollii, as well as representatives [wuc] of the Pleosporales order and Didymellaceae family. Although there are no descriptions of dysbioses in the literature associating $T$. stollii with any type of disease, 95 detected a relative reduction in representatives of the genus Talaromyces in mice submitted to hypercaloric diets, while the species Talaromyces islandicus was positively correlated with the development of CRC 
(46). Members [wuc] of the Pleosporales order, however, have been associated with healthy mucosa in studies involving the occurrence of UV light-derived eye inflammation (96), as well as in the study on CD described by 82 .

On the other hand, representatives of the genus Malassezia, the only Basidiomycota whose populations have been shown to expand in the gut of CC mice, have a long history of correlation with dysbioses associated with skin inflammation and dermatological diseases, since they are the main mycobiota components of the human and animal skin $(97,98,99,100)$. Although the location of these fungi in the digestive tract has been previously described, the acceptance of these microorganisms as permanent inhabitants of the intestinal mycobiota is still a cause for debate (101). Nonetheless, 83 found a decrease in the relative population of Malasseziales in the intestine of patients affected by CD, while these same microorganisms were increased in the intestine of UC patients, suggesting that these fungi could be used as biomarkers to differentiate these two forms of IBD. More recently, 102 demonstrated that the species $M$. rescricta exacerbates symptoms of colitis in gnotobiotic mice and is present at a greater proportion in the colonic mycobiota of human subjects affected by $\mathrm{CD}$, particularly in individuals with a specific polymorphism in the CARD9 gene. This gene encodes a protein responsible for recruiting immune cells to fight fungal infections and, curiously, patients affected by CD are known to develop anti-CARD9 antibodies (102). Malassezia representatives also compose a significant part of the microorganisms that invade the pancreatic duct, from the intestinal lumen, contributing to the development of Pancreatic Ductal Adenocarcinoma (PDA), through activation of the complement cascade, via interaction with mannan-binding lectins (MBLs) (103, 104). More recently, increases in the relative proportion of Malassezia have also been found in the gut of CRC patients and of children showing autoimmunity against pancreatic beta cells, which later developed type 1 diabetes $(46,105)$.

The cachexia-associated fungal dysbiosis described herein is also characterized by additional fungal groups, whose relative proportions are decreased in CC animals (Figure 4). These include members of the Cystobasidiomycetes class (with emphasis on the species Cystobasidium sloofiae) and representatives [wuc] of the Sporidiobolaceae family. Although there are few reports regarding the involvement of these fungal groups with diseaseassociated dysbioses, increased populations of Sporidiobolaceae have been detected in cervical mucosa lesions from HPV-infected women at low risk of malignancy (106), as well as in the intestine of mice submitted to diets rich in animal proteins (107). Moreover, the relative abundance of Cystobasidiomycetes was positively correlated with adiposity, while negatively correlated with the serum concentration of LDL cholesterol in obese and lean subjects (69).

As mentioned above, obesity-associated fungal dysbiosis differs from the pattern observed in $\mathrm{CRC} / \mathrm{ME} / \mathrm{IBD} / \mathrm{UC} / \mathrm{CD}$, as no significant changes have been detected in the relative ratio of Ascomycota/Basidiomycota, when obese individuals are compared to lean ones. In fact, the main characteristic found in obesity-associated mycobiota refers to a reduction in the proportion of Mucoromycota, a pattern also observed in our CC-associated dysbiosis (Figures 3 and 4). Thus, the CC-related dysbiosis seems to be unique, presenting features observed both in obesity (reduced proportion of Mucoromycota) and $\mathrm{CRC} / \mathrm{ME} / \mathrm{IBD} / \mathrm{UC} / \mathrm{CD}$, with increased proportions of different groups of Ascomycota (such as Sordariomycetes and Saccharomycetaceae), as well as Malassezia.

Finally, it is worth mentioning that the population analysis shown in Figure 4 allowed identification of four fungal species preferably present in the gut of SC animals, which can be grown under axenic laboratory conditions (Rhizopus oryzae, Cystobasidium 
sloofiae, Penicillium citrinum and Cladiosporium halotolerans). These fungi are natural candidates to be tested (individually, or in combinations) for their eventual roles as probiotic agents, aiming at the prevention/treatment of cachexia $(76,108)$. Rhyzopus oryzae, in particular, stands out as the most promising probiotic candidate, not only due to its capacity to produce bioactive substances, such as chitosan and gallic acid, but also to its historical use as a component of traditional oriental foods, which has granted it the status of a GRAS (Generally Recognized As Safe) filamentous fungus, according to the US Food and Drug Administration (FDA) (76).

\section{ACKNOWLEDGEMENTS}

This study was financed in part by the São Paulo Research Foundation-FAPESP (http://www.fapesp.br) Grants \#2017/13197-8 and 2017/08112-3, awarded to L.R.N and D.L.J. Y.N.L.F.M., F.B.M., D.A.B., K.B.N.H.S. and V.C.A. are recipients of scholarship grants from Coordenação de Aperfeiçoamento de Pessoal de Nível Superior - Brasil CAPES (http://www.capes.gov.br/). L.M.C. and A.C.H are recipients of scholarship grants from Conselho Nacional de Desenvolvimento Científico e Tecnológico - Brasil - CNPq. Authors would like to thank the Core Facility for Scientific Research - University of São Paulo (CEFAP-USP/GENIAL [Genome Investigation and Analysis Laboratory]) for the Bioanalyzer 2100 services.

\subsection{REFERENCES}

1. Evans, W. J., et al. (2008). Cachexia: A new definition. Clinical Nutrition, 27(6), 793-799.

2. Fearon, K., et al. (2011). Definition and classification of cancer cachexia: an international consensus. The Lancet Oncology, 12(5), 489-495.

3. Bing, C., \& Trayhurn, P. (2008). Regulation of adipose tissue metabolism in cancer cachexia. Current Opinion in Clinical Nutrition and Metabolic Care, 11(3), 201-207.

4. Gordon, J. N., Green, S. R., \& Goggin, P. M. (2005). Cancer cachexia. QJM: An International Journal of Medicine, 98(11), 779-788.

5. Bruera, E., \& Hui, D. (2012). Conceptual models for integrating palliative care at cancer centers. Journal of palliative medicine, 15(11), 1261-1269.

6. Bindels, L. B., et al. (2012a). Restoring Specific Lactobacilli Levels Decreases Inflammation and Muscle Atrophy Markers in an Acute Leukemia Mouse Model. PLoS ONE, 7(6), e37971.

7. Bindels, L.B., et al. (2012b). Gut microbiota-derived propionate reduces cancer cell proliferation in the liver. Br J Cancer, 107(8):1337-1344.

8. Bindels, L., et al. (2015a). SUN-PP131: Synbiotic intervention improves intestinal homeostasis, cachexia and survival in leukemic mice with cachexia. Clinical Nutrition, 34, S72.

9. Bindels, L. B., Walter, J. \& Ramer-Tait, A. E. (2015b). Resistant starches for the management of metabolic diseases. Current opinion in clinical nutrition and metabolic care, 18(6), 559-565.

10. Bindels, L. B., et al. (2016). Synbiotic approach restores intestinal homeostasis and prolongs survival in leukaemic mice with cachexia. The ISME journal, 10(6), 1456-1470.

11. Bindels, L. B., et al. (2018). Increased gut permeability in cancer cachexia: mechanisms and clinical relevance. Oncotarget, 9(26), 18224-18238. 
12. Elinav, E., et al. (2011). NLRP6 inflammasome regulates colonic microbial ecology and risk for colitis. Cell, 145(5), 745-757. https://doi.org/10.1016/j.cell.2011.04.022

13. Plunkett, C. H. \& Nagler, C. R. (2017). The Influence of the Microbiome on Allergic Sensitization to Food. Journal of immunology (Baltimore, Md.: 1950), 198(2), 581-589.

14. Tözün, N. \& Vardareli, E. (2016). Gut Microbiome and gastrointestinal cancer. Journal of Clinical Gastroenterology, 50, S191-S196 4.

15. Qin, Q., et al. (2012). Normal and disease-related biological functions of Twist1 and underlying molecular mechanisms. Cell research, 22(1), 90-106.

16. Karlsson, F. H., et al. (2013). Gut metagenome in European women with normal, impaired and diabetic glucose control. Nature, 498(7452), 99-103.

17. Ley, R. E., et al. (2005). Obesity alters gut microbial ecology. Proceedings of the National Academy of Sciences of the United States of America, 102(31), 11070-11075.

18.Turnbaugh, P. J., et al. (2006). An obesity-associated gut microbiome with increased capacity for energy harvest. Nature, 444(7122), 1027-1031.

19. Verdam, F. J., et al. (2013). Human intestinal microbiota composition is associated with local and systemic inflammation in obesity. Obesity, 21(12), E607-E615.

20. Buttó, L. F., \& Haller, D. (2017). Functional relevance of microbiome signatures: The correlation era requires tools for consolidation. Journal of Allergy and Clinical Immunology, 139(4), 10921098..

21. Pötgens, S.A., et al. (2018). Klebsiella oxytoca expands in cancer cachexia and acts as a gut pathobiont contributing to intestinal dysfunction. Sci Rep.,17,8(1), 12321.

22. Ebner, N., Anker, S. D., \& Haehling, S. (2019). Recent developments in the field of cachexia, sarcopenia, and muscle wasting: highlights from the 11th Cachexia Conference. Journal of Cachexia, Sarcopenia and Muscle, 10(1), 218-225.

23. Henriques, F. S., et al. (2017). Early suppression of adipocyte lipid turnover induces immunometabolic modulation in cancer cachexia syndrome. The FASEB Journal, 31(5), 19761986.

24. Voltarelli, F. A., et al. (2017). Syngeneic B16F10 Melanoma Causes Cachexia and Impaired Skeletal Muscle Strength and Locomotor Activity in Mice. Front. Physiol., 8.

25. Batista, M. L., Jr, et al. (2016). Cachexia-associated adipose tissue morphological rearrangement in gastrointestinal cancer patients. Journal of cachexia, sarcopenia and muscle, 7(1), 37-47.

26. Kemik, O., et al. (2010). The relationship among acute-phase response proteins, cytokines and hormones in cachectic patients with colon cancer. World journal of surgical oncology, 8, 85.

27. Zhou, X., et al. (2010). Reversal of Cancer Cachexia and Muscle Wasting by ActRIIB Antagonism Leads to Prolonged Survival. Cell, 142(4), 531-543.

28. Batista, M. L., Jr., et al. (2013). Adipose tissue-derived factors as potential biomarkers in cachectic cancer patients. Cytokine, 61(2), 532-539.

29. Cui, L., Morris, A., \& Ghedin, E. (2013). The human mycobiome in health and disease. Genome medicine, 5(7), 63.

30. Ewels, P., et al. (2016). MultiQC: summarize analysis results for multiple tools and samples in a single report. Bioinformatics, 32(19), 3047-3048.

31. Edgar, R. C. (2010). Search and clustering orders of magnitude faster than BLAST. Bioinformatics, 26(19), 2460-2461. 
32. Caporaso, J. G., et al. (2010). QIIME allows analysis of high-throughput community sequencing data. Nature Methods, 7(5), 335-336.

33. DeSantis, T. Z., et al. (2006). Greengenes, a Chimera-Checked 16S rRNA Gene Database and Workbench Compatible with ARB. Applied and Environmental Microbiology, 72(7), 50695072 .

34. Nilsson, R. H., et al. (2019). The UNITE database for molecular identification of fungi: handling dark taxa and parallel taxonomic classifications. Nucleic acids research, 47(D1), D259-D264.

35. Kopylova, E., Noé, L., \& Touzet, H. (2012). SortMeRNA: fast and accurate filtering of ribosomal RNAs in metatranscriptomic data. Bioinformatics, 28(24), 3211-3217.

36. Mercier, C., et al. (2013). SUMATRA and SUMACLUST: fast and exact comparison and clustering of sequences. In: Programs and Abstracts of the SeqBio 2013 workshop (Abstract), GdRBIM and gdrIM, Montpellier, France. 27-29. Available at http://metabarcoding.org/ sumatra.

37. Wang, Q., et al. (2007). Naive Bayesian classifier for rapid assignment of rRNA sequences into the new bacterial taxonomy. Applied and environmental microbiology, 73(16), 5261-5267.

38. Lefevre, E., et al. 2016). Characterization and Adaptation of Anaerobic Sludge Microbial Communities Exposed to Tetrabromobisphenol A. PloS One, 11(7), e0157622.

39. Kluyver, T., et al. (2016). Jupyter Notebooks \&ndash, a publishing format for reproducible computational workflows. Stand Alone, 0(Positioning and Power in Academic Publishing: Players, Agents and Agendas), 87-90.

40. Menegidio, F. B., et al. (2018). Dugong: a Docker image, based on Ubuntu Linux, focused on reproducibility and replicability for bioinformatics analyses. Bioinformatics, 34(3), 514-515.

41. Menegidio, F. B. et al. (2019). Bioportainer Workbench: a versatile and user-friendly system that integrates implementation, management, and use of bioinformatics resources in Docker environments. GigaScience, 8(4).

42. Dhariwal, A. et al. (2017). MicrobiomeAnalyst: a web-based tool for comprehensive statistical, visual and meta-analysis of microbiome data. Nucleic Acids Research, 45(W1), W180W188.43.

43. Chong, J. et al. (2020). Using MicrobiomeAnalyst for comprehensive statistical, functional, and meta-analysis of microbiome data. Nature Protocols, 799-821.

44. Beals, E. W. (1984). Bray-Curtis Ordination: An Effective Strategy for Analysis of Multivariate Ecological Data. In Advances in Ecological Research (pp. 1-55). Elsevier.

45. Anderson, M.J. (2001). A new method for non $\square$ parametric multivariate analysis of variance. Aust Ecol., 26, 32-46.

46. Coker, O. O. et al. (2019). Enteric fungal microbiota dysbiosis and ecological alterations in colorectal cancer. Gut., 68, 654-662.

47. Cameron, S.J. et al. (2016). Metagenomic Sequencing of the Chronic Obstructive Pulmonary Disease Upper Bronchial Tract Microbiome Reveals Functional Changes Associated with Disease Severity. PLoS One. 2016;11(2):e0149095.

48. Astudillo-García, C. et al. (2017). Evaluating the core microbiota in complex communities: A systematic investigation. Environmental Microbiology, 19(4), 1450-1462.

49. Flemer, B, et al. (2017). Fecal microbiota variation across the lifespan of the healthy laboratory rat. Gut Microbes.,8(5):428-439. 
50. Björk, J. R. et al. (2017). The dynamic core microbiome: Structure, dynamics and stability. Cold Spring Harbor Laboratory. bioRxiv; 2017.

51. Toju, H. et al. (2018). Core microbiomes for sustainable agroecosystems [published correction appears in Nat Plants. 2018 Sep;4(9):733]. Nat Plants. 2018;4(5):247-257.

52. Russel, J. et al. (2018). Genetic Risk for Type 1 Diabetes Profoundly Influences the Core Gut Microbiome in Children. Diabetes, 67(Supplement 1), 209-LB.

53. Piampiano, E. et al. (2019). Analysis of microbiota in cultures of the green microalga Tetraselmis suecica. European Journal of Phycology, 54:3, 497-508.

54. Suenami, S. et al. (2019). Community analysis of gut microbiota in hornets, the largest eusocial wasps, Vespa mandarinia and V. simillima. Scientific reports, 9(1), 9830.

55. Wallace, R.J. et al. (2019). A heritable subset of the core rumen microbiome dictates dairy cow productivity and emissions. Sci Adv. 2019;5(7):eaav8391.

56. Clos-Garcia, M. et al. (2019). Gut microbiome and serum metabolome analyses identify molecular biomarkers and altered glutamate metabolism in fibromyalgia. EBioMedicine 2019;46:499-511.

57. Mallick, H. et al. (2017). Experimental design and quantitative analysis of microbial community multiomics. Genome biology, 18(1), 228.

58. Segata, N. et al. (2011). Metagenomic biomarker discovery and explanation. Genome biology, 12(6), R60.

59. Dolatabadi S. et al. (2014) Species boundaries and nomenclature of Rhizopus arrhizus (syn. R. oryzae). Mycoses, 57 (3),108-27.

60. Sugita, T. et al. (2003). Description of a New Yeast Species, Malassezia japonica, and Its Detection in Patients with Atopic Dermatitis and Healthy Subjects. Journal of clinical microbiology., 41,4695-4699.

61. Nagao, K. et al. (2005). Genetic identification and detection of human pathogenic Rhizopus species, a major mucormycosis agent, by multiplex PCR based on internal transcribed spacer region of rRNA gene. J Dermatol Sci.,39(1),23-31.

62. Chang, H.W. et al. (2007). Quantitative real time PCR assays for the enumeration of Saccharomyces cerevisiae and the Saccharomyces sensu stricto complex in human feces. J Microbiol Methods, 71(3), 191-201.

63. Hierro, N., et al. (2007). Monitoring of Saccharomyces and Hanseniaspora populations during alcoholic fermentation by real-time quantitative PCR (2007). FEMS Yeast Res., 7(8), 1340-9.

64. Vuran, E. et al. (2014). Identification of Malassezia species from pityriasis versicolor lesions with a new multiplex PCR method. Mycopathologia. 2014 Feb,177(1-2):41-9.

65. Xie, Z et al. (2014). An Analysis of the Malassezia Species Distribution in the Skin of Patients with Pityriasis Versicolor in Chengdu, China. TheScientific WorldJournal, 182596.

66. Urubschurov, V. et al. (2015). Development and Evaluation of qPCR Assay for Quantitation of Kazachstania slooffiae and Total Yeasts Occurring in the Porcine Gut. Current Microbiology, 432(71), 373-281.

67. McKernan, K. et al. (2016) Cannabis microbiome sequencing reveals several mycotoxic fungi native to dispensary grade Cannabis flowers. F1000Research, 4, 1422.

68. Bajaj, J.S. et al. (2018). Fungal dysbiosis in cirrhosis. Gut,67(6),1146-1154.

69. Mar Rodríguez, M. et al. (2015).Obesity changes the human gut mycobiome. Sci Rep., 5, 14600. 
70. Sumiyoshi, M., \& Kimura, Y. (2006). Low molecular weight chitosan inhibits obesity induced by feeding a high-fat diet long-term in mice. The Journal of pharmacy and pharmacology, 58(2), 201-207.

71. Walsh, A. M. et al. (2013). Multi-functional roles of chitosan as a potential protective agent against obesity. PloS one, 8(1), e53828.

72. Cardoso, A. et al. (2012). Microbial enhance of chitosan production by Rhizopus arrhizus using agroindustrial substrates. Molecules (Basel, Switzerland), 17(5), 4904-4914.

73. Tasar, O. C., Erdal, S., \& Taskin, M. (2016). Chitosan production by psychrotolerant Rhizopus oryzae in non-sterile open fermentation conditions. International journal of biological macromolecules, 89, 428-433.

74. Sebastian, J. et al. (2019). Microwave-assisted extraction of chitosan from Rhizopus oryzae NRRL 1526 biomass. Carbohydrate polymers, 219, 431-440.

75. Wang, W. et al. (2020). Chitosan Derivatives and Their Application in Biomedicine. International journal of molecular sciences, 21(2), 487.

76. Londoño-Hernández, L. et al. (2017). Rhizopus oryzae - Ancient microbial resource with importance in modern food industry. International journal of food microbiology, 257, 110-127.

77. Sambanthamurthi, R. et al. (2011). Positive outcomes of oil palm phenolics on degenerative diseases in animal models. The British journal of nutrition, 106(11), 1664-1675.

78. Leow, S. S. et al. (2013). Gene expression changes in spleens and livers of tumour-bearing mice suggest delayed inflammation and attenuated cachexia in response to oil palm phenolics. Journal of nutrigenetics and nutrigenomics, 6(6), 305-326.

79. Bak, E. J. et al. (2013). Gallic acid improves glucose tolerance and triglyceride concentration in diet-induced obesity mice. Scandinavian journal of clinical and laboratory investigation, 73(8), 607-614.

80. Dludla, P. V. et al. (2018). Inflammation and Oxidative Stress in an Obese State and the Protective Effects of Gallic Acid. Nutrients, 11(1), 23.

81. Maresca, M. \& Fantini, J. (2010). Some food-associated mycotoxins as potential risk factors in humans predisposed to chronic intestinal inflammatory diseases. Toxicon.,56(3),282-94.

82. Liguori, G. et al. (2016). Fungal Dysbiosis in Mucosa-associated Microbiota of Crohn's Disease Patients. J Crohns Colitis, 10(3),296-305.

83. Sokol, H. et al. (2017). Fungal microbiota dysbiosis in IBD. Gut, 66(6), 1039-1048.

84. Mandarano, A. H. et al. (2018). Eukaryotes in the gut microbiota in myalgic encephalomyelitis/chronic fatigue syndrome. PeerJ, 6:e4282.

85. Luan, C. et al. (2015). Dysbiosis of fungal microbiota in the intestinal mucosa of patients with colorectal adenomas. Sci. Rep., 5, 7980.

86. Antonissen, G. et al. (2014). The impact of Fusarium mycotoxins on human and animal host susceptibility to infectious diseases. Toxins (Basel), 6(2):430-52.

87. Maresca, M. (2013). From the gut to the brain: journey and pathophysiological effects of the food-associated trichothecene mycotoxin deoxynivalenol. Toxins (Basel), 5(4),784-820. 88.

86. Kurtzman, C. P., et al. (005).Multigene phylogenetic analysis of pathogenic candida species in the Kazachstania (Arxiozyma) telluris complex and description of their ascosporic states as Kazachstania bovina sp. nov., $K$. heterogenica sp. nov., $K$. pintolopesii sp. nov., and $K$. slooffiae sp. nov. J Clin Microbiol.,43(1):101 $\square 111$. 
89. Arfken, A.M. et al. (2019). Yeasts of Burden: Exploring the Mycobiome-Bacteriome of the Piglet GI Tract. Front Microbiol.,10, 2286.

90. Jawhara, S. et al. (2012). Modulation of intestinal inflammation by yeasts and cell wall extracts: Strain dependence and unexpected anti-inflammatory role of glucan fractions. PLoS One, 7 (2012), e40648.

91. Chiaro, T.R et al. (2017). A member of the gut mycobiota modulates host purine metabolism exacerbating colitis in mice. Sci Transl Med., 9, 380.

92. Lamprinaki, D. et al. (2017). LC3-Associated Phagocytosis Is Required for Dendritic Cell Inflammatory Cytokine Response to Gut Commensal Yeast Saccharomyces cerevisiae. Front Immunol.,8,1397.

93. Giaffer, M. H., Clark, A., Holdsworth, C. D. (1992). Antibodies to Saccharomyces cerevisiae in patients with Crohn's disease and their possible pathogenic importance. Gut, 33(8), 1071-1075.

94. Mitsuyama, K. et al. (2016). Antibody markers in the diagnosis of inflammatory bowel disease. World J Gastroenterol.,22(3),1304-10.

95. Van der Merwe, M. et al. (2020). Time of Feeding Alters Obesity-Associated Parameters and Gut Bacterial Communities, but Not Fungal Populations, in C57BL/6 Male Mice. Current Developments in Nutrition, 4(2).

96. Jayasudha, R. et al. (2019). Implicating Dysbiosis of the Gut Fungal Microbiome in Uveitis, an Inflammatory Disease of the Eye. Invest Ophthalmol Vis Sci., 60(5), 1384-1393.

97. Malassez, L. (1874). Note sur le champignon du pityriasis simple. Archives de Physiologie Normale et Pathologique, 2:451-464.

98. Xu, J. et al. (2007). Dandruff-associated Malassezia genomes reveal convergent and divergent virulence traits shared with plant and human fungal pathogens. PNAS, 104 (47), 18730-18735.

99. Soares, R.C. et al. (2015). Malassezia intra-specific diversity and potentially new species in the skin microbiota from Brazilian healthy subjects and seborrheic dermatitis patients. PLoS One, 10(2), 0117921.

100.Velegraki, A. et al. (2015). Malassezia Infections in Humans and Animals: Pathophysiology, Detection, and Treatment. PLoS Pathog., 11(1), e1004523.

101. Hallen-Adams, H.E., Suhr, M.J. Fungi in the healthy human gastrointestinal tract (2017). Virulence, 8, 352-8.

102. Limon, J. J. et al. (2019). Malassezia Is Associated with Crohn's Disease and Exacerbates Colitis in Mouse Models. Cell Host Microbe., 25(3), 377-388.e6.

103. Aykut, B. et al. (2019). The fungal mycobiome promotes pancreatic oncogenesis via activation of MBL. Nature, 574(7777), 264-267.

104. Van Asbeck, E. C. et al. (2008). Mannose binding lectin plays a crucial role in innate immunity against yeast by enhanced complement activation and enhanced uptake of polymorphonuclear cells. BMC Microbiol., 8, 229.

105. Honkanen, J. et al. (2020) Fungal Dysbiosis and Intestinal Inflammation in Children With BetaCell Autoimmunity. Front. Immunol., 11, 468.

106. Godoy-Vitorino, F. et al. (2018). Cervicovaginal fungi and bacteria associated with cervical intraepithelial neoplasia and high-risk human papillomavirus infections in a Hispanic population. Front.Microb., 9, 2533. 
bioRxiv preprint doi: https://doi.org/10.1101/2020.06.29.171397; this version posted July 3, 2020. The copyright holder for this preprint (which was not certified by peer review) is the author/funder, who has granted bioRxiv a license to display the preprint in perpetuity. It is made available under aCC-BY-ND 4.0 International license.

107. Kostovcikova, K. et al. (2019) Diet Rich in Animal Protein Promotes Pro-inflammatory Macrophage Response and Exacerbates Colitis in Mice. Front. Immunol., 10, 919.

108. da Costa, S. G. et al. (2018). Penicillium citrinum UFV1 $\beta$-glucosidases: purification, characterization, and application for biomass saccharification. Biotechnol Biofuels,11,226. 


\subsection{FIGURE LEGENDS}

Figure 1 - Characterization of cachexia in the animals selected for this study. A group of 16 animals was selected for the analyses described herein. Eight (8) of them received saline injection (SC group), while others were injected with LLC cells (CC group). After 28 days, the animals were evaluated for a series of characteristics typically associated with cachexia. Panel A shows the average weight gain of the SC and CC animals 28 days after injection (tumor weight subtracted), as well as the Cachexia Indexes (CI) among CC animals. Panels $\mathrm{B}$ and $\mathrm{C}$ show that the $\mathrm{CC}$ animals also showed increased relative expression of muscular atrophy and inflammation markers (Atrogin and IL6R, respectively), as verified by qPCR. Panel D shows examples of histological analyses made with epididymal adipose tissue (EPI) (top) and gastrocnemius muscle (GAS) (bottom) obtained from SC (left) and CC (right) animals, demonstrating significant reduction in the average adipocyte size and relative area of muscular fibers in CC animals. These reductions are confirmed by histometric evaluation of representative cross sections made with all animals from each group (Panel E). Magnification of adipose tissue: 20x; magnification of muscle tissue: $10 x ; *=p \leq 0.05$, after $\mathrm{T}$ test. Results for SC animals are shown in blue, while results for $\mathrm{CC}$ animals are shown in red.

Figure 2 - Alpha and Beta diversity analyses comparing the mycobiota of SC and CC animals. Panel A shows the results of alpha diversity analysis (absolute number of fungi). The OTU Table obtained from the sequences of ITS1 amplicons was filtered to keep only OTUs with abundance above 0.00001 and used to calculate the absolute number of fungi present in the stool samples from SC and CC animals, at different taxonomic levels. The figure shows the results obtained for the levels of species (left) and OTU (right). In both cases, it was not possible to verify significant changes in fungal alpha diversity between the two groups, since p-values obtained from such analyses, after T test, were always above 0.05. Similar results (not shown) were obtained with other alpha diversity metrics and/or after extending these analyses to additional taxonomy levels (see text for details). Panel B shows the result of a Beta-diversity analysis to evaluate differences in composition between the fungal populations present in the stool samples from SC and CC animals. This analysis was performed with the aid of a Non-linear Multidimensional Scaling (NMDS) algorithm (based on the Bray-Curtis index), using the same filtered OTU Table described above. The NMDS analysis shows that differences in mycobiota composition allow statistically significant discrimination between the $\mathrm{SC}$ and $\mathrm{CC}$ groups (at $\mathrm{p}=0.045$, as verified by PERMANOVA), although the two main components of the NMDS analysis do not allow us to visualize such distinction. Animals from the SC group are shown in blue, while animals from the $\mathrm{CC}$ group are shown in red.

Figure 3 - Population composition of the mycobiota from SC and CC animals and evaluation of fungal Dysbiosis Ratios (DR) associated with the development of cachexia. The OTU Table obtained from the sequences of ITS1 amplicons was filtered to keep only OTUs with abundance above 0.00001 and used to calculate the relative proportions of the main fungal phyla present in the gut of SC and $\mathrm{CC}$ animals. Panel A shows that the gut mycobiota of SC animals are mostly represented by Ascomycota $(\sim 85.1 \%)$, followed by Basidiomycota $(\sim 13.3 \%)$ and Mucoromycota $(\sim 1.5 \%)$. These proportions are shifted in the gut mycobiota of $\mathrm{CC}$ animals to $\sim 87.2 \%$ (Ascomycota), 
$\sim 12.5 \%$ (Basidiomycota) and $\sim 0.4 \%$ (Mucoromycota). These values were used to calculate the Dysbiosis Ratios (DR) of SC and CC animals, involving the relative proportions of MucoromycotalAscomycota (Panel B), MucoromycotalBasidiomycota (Panel C) and BasidiomycotalAscomycota (Panel D). Results for SC animals are shown in blue, while results for CC animals are shown in red $\left(^{*}=\mathrm{p} \leq 0.05\right.$, after Mann-Whitney U test).

Figure 4 - Identification of the main fungal taxa differentially represented in stool samples from SC and CC animals. The OTU Table obtained from the sequences of ITS1 amplicons was initially filtered to keep only OTUs with abundances above 0.00001 and present in at least $70 \%$ of the animals within each group, minimizing the effect of taxa overrepresented in few individuals within a group, but not consistently present throughout the group. The Core 70 OTU Table derived from these filtration procedures was subjected to a LEfSe analysis, using $\mathrm{p} \leq 0.05$ and a modular limit for LDA Score $\geq 2(-2 \leq$ LDA Score $\geq 2)$ as stringency criteria to identify differentially represented taxa between CC and SC animals, respectively. All differentially represented taxa (at different taxonomic levels) are shown in the left panel of the figure and their distribution in a phylogenetic dendrogram is shown at the center panel. The concentric circles of the dendrogram show the taxonomic hierarchy, from phylum (innermost circle) to species (outermost circle) and the different microorganisms distributed in each node can be identified with the aid of the legend shown on the right panel. Microorganisms overrepresented in stool samples from SC animals are shown in blue, while microorganisms overrepresented in stool samples from CC animals are shown in red.

Figure 5 - Relative quantification of different microorganisms in stool samples from SC and CC animals by qPCR. Equimolar samples of DNA extracted from stool samples obtained from each mouse were consolidated into two pools, representing SC and CC groups. Samples from these pools were then used in qPCR experiments, with primers previously described in the literature as specific to Saccharomyceae, Kazachstania and Malassezia genera, as well as to the species Saccharomyces cereviseae, Malassezia dermatis, Malassezia japonica, Rhizopus oryzeae and Penicillium citrinum. The $C t$ values obtained for each of these taxa were normalized by the $C t$ obtained after amplifying the pooled DNAs with the primer pair used to amplify the fungal ITS1 amplicons. Next, the normalized $C t \mathrm{~s}$ were used to calculate the relative prevalence of each genus/species in the $\mathrm{CC}$ pool, in relation to the SC pool (CC/CR). The relative quantification values for each taxon (Fold Change) are shown in the graph, as the mean \pm standard deviation of three independent experiments (each performed in triplicate). The species Rhizopus oryzae was detected only in the DNA pool from SC animals, so its SC/CC ratio is represented in the graph as negative, to infinity (-INF). The species Penicillium citrinum was not detected (N/D) in any of the experiments, with either DNA pool. 


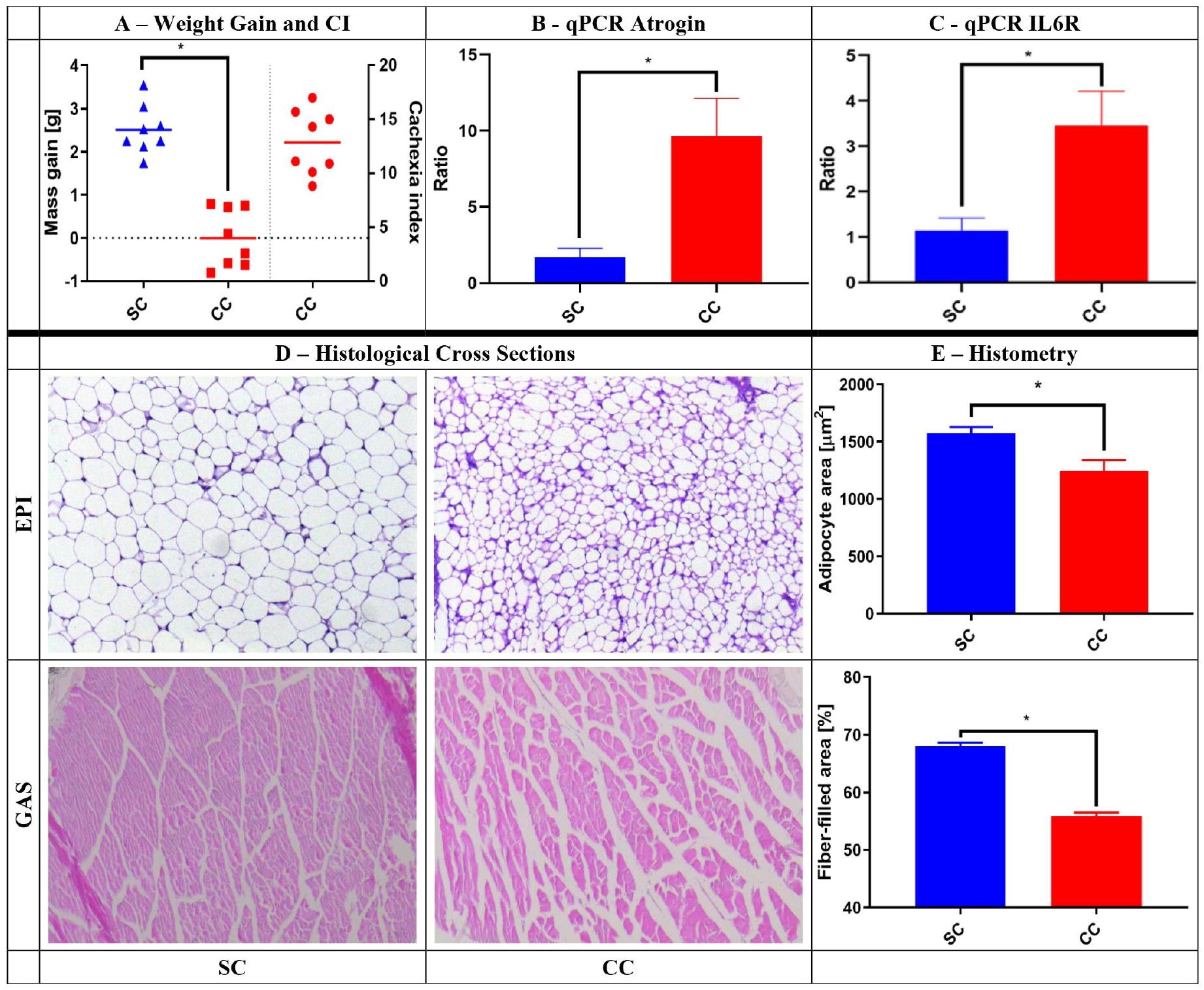




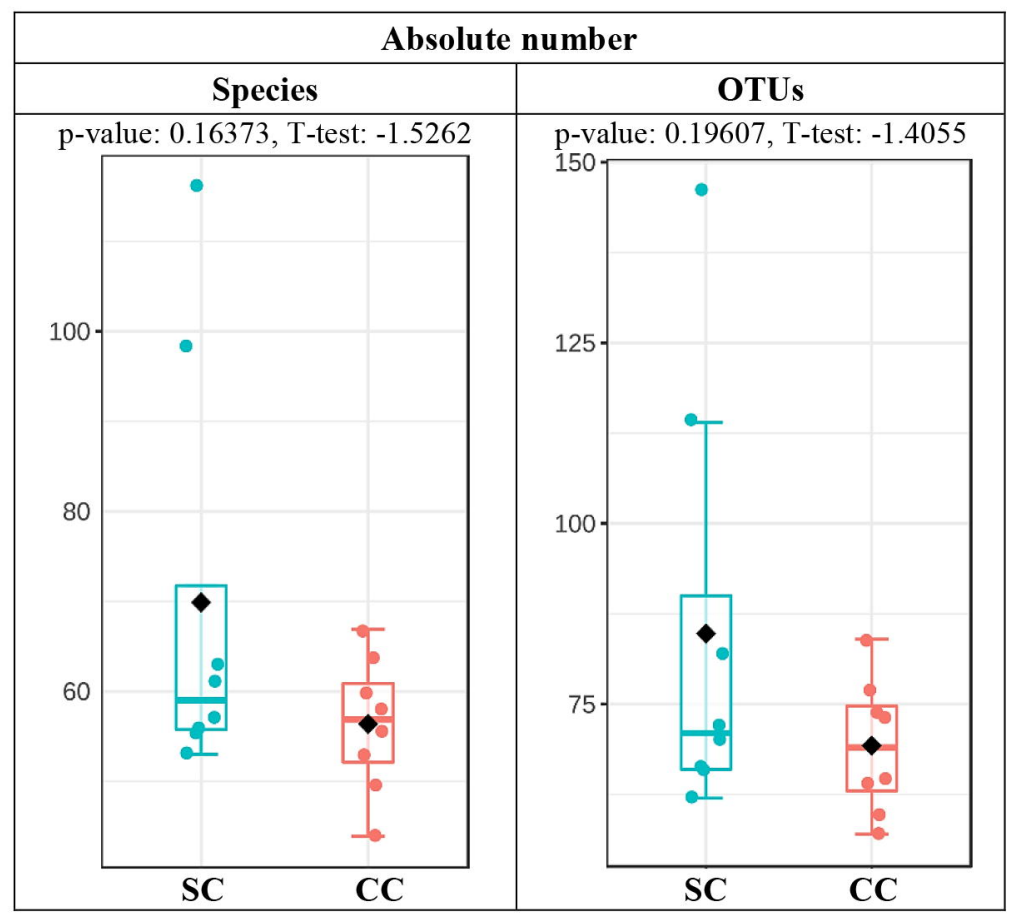

B

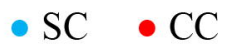

PERMANOVA: $\mathrm{F}=2.7845 ; \mathrm{R}^{2}=0.1659 ; \mathrm{p}$-value $=0.045 ;$ NMDS Stress: 0.18772

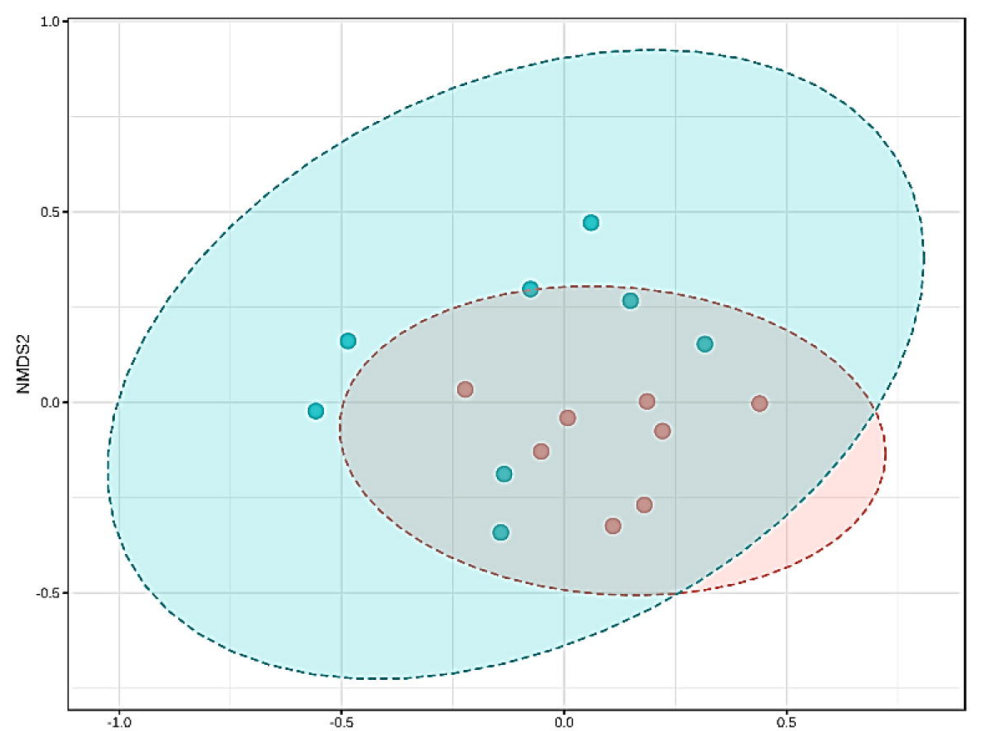



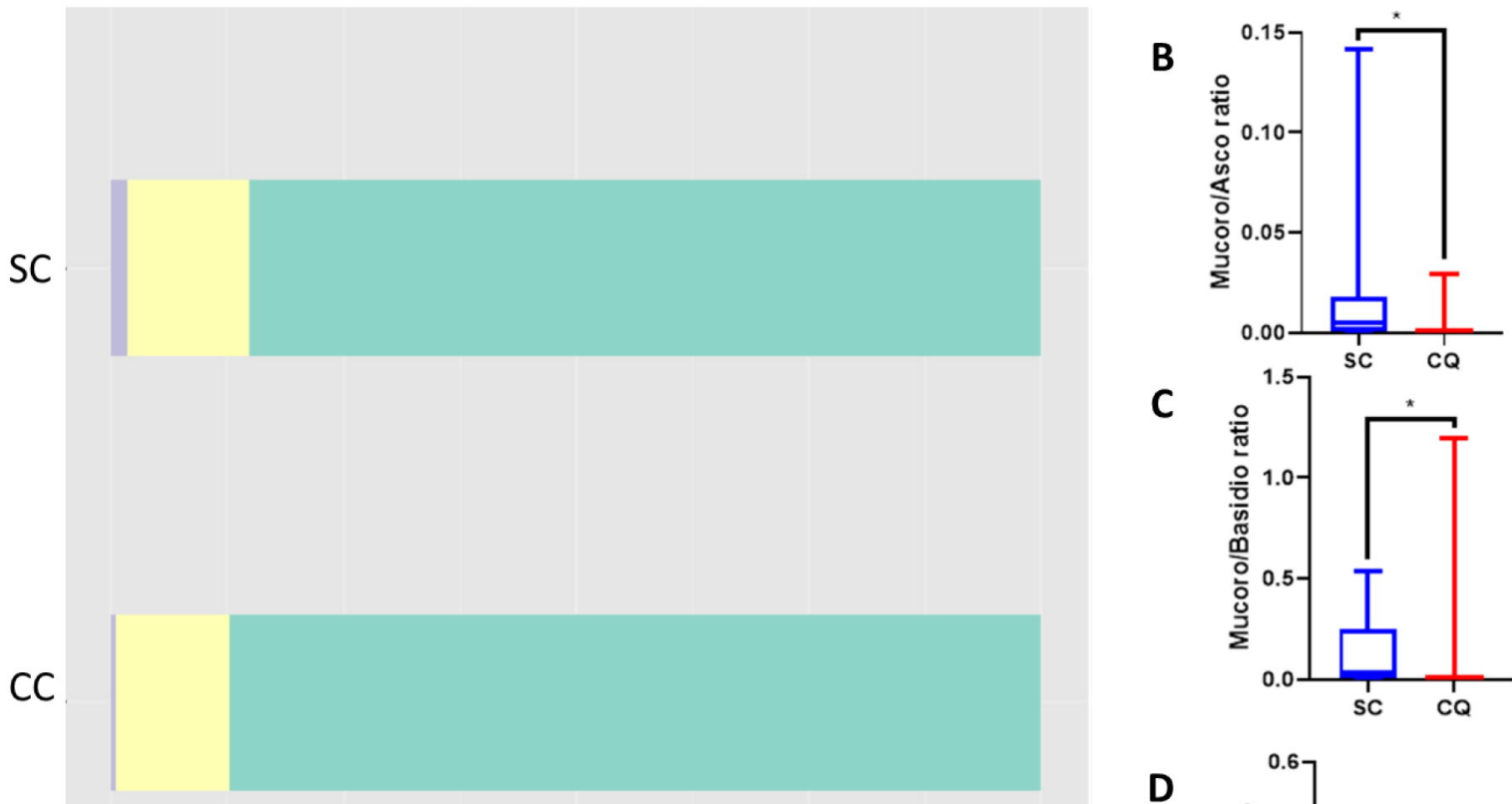

0.00

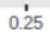

C

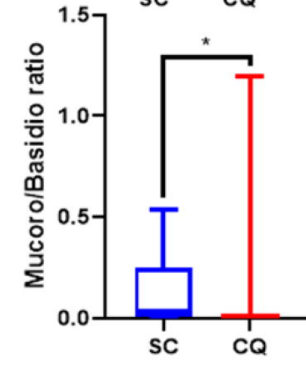

D

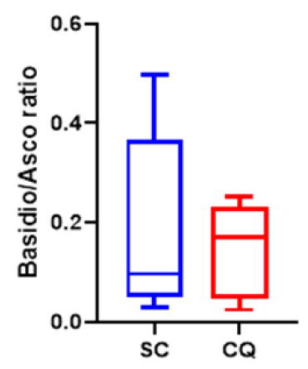



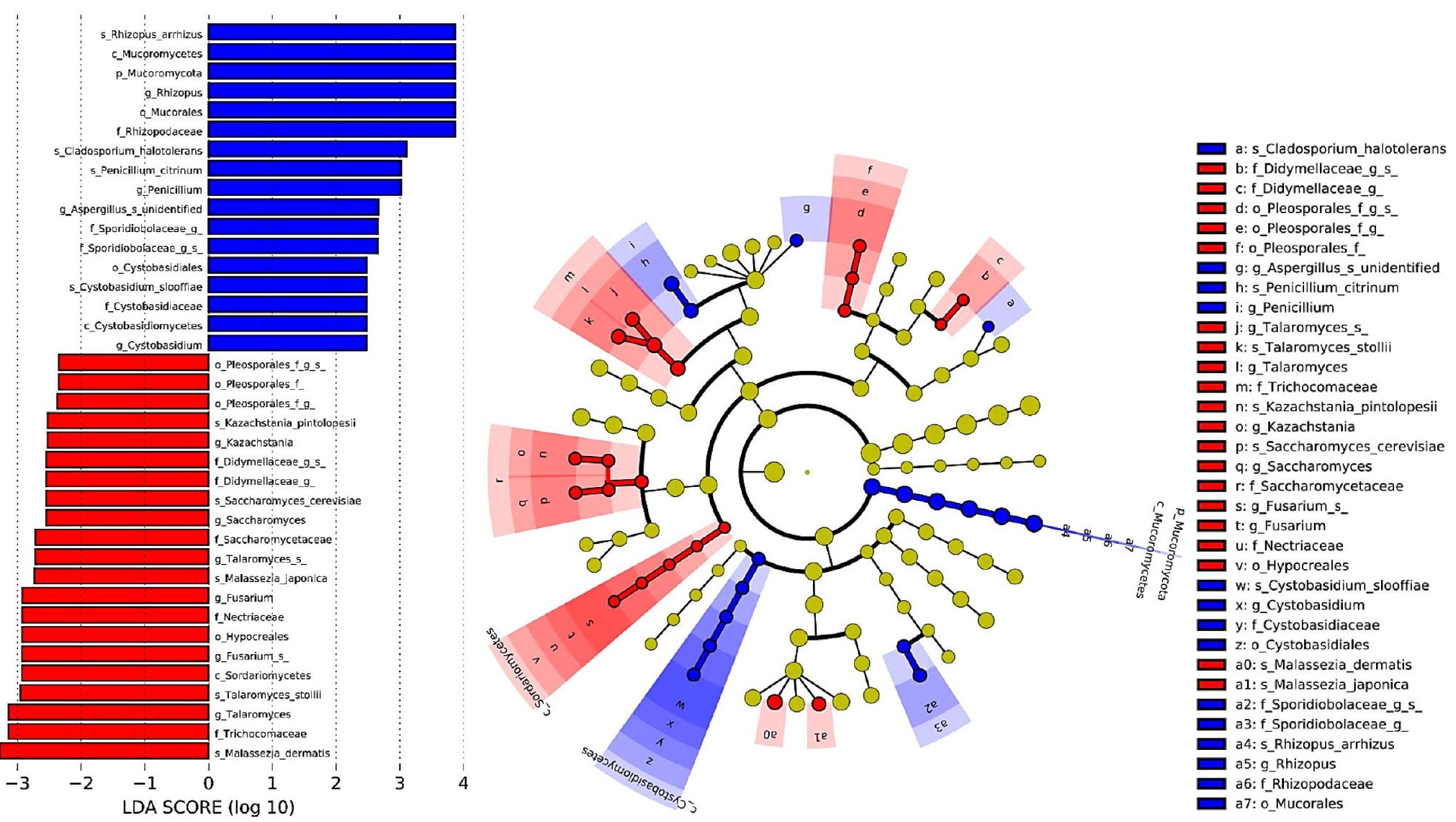


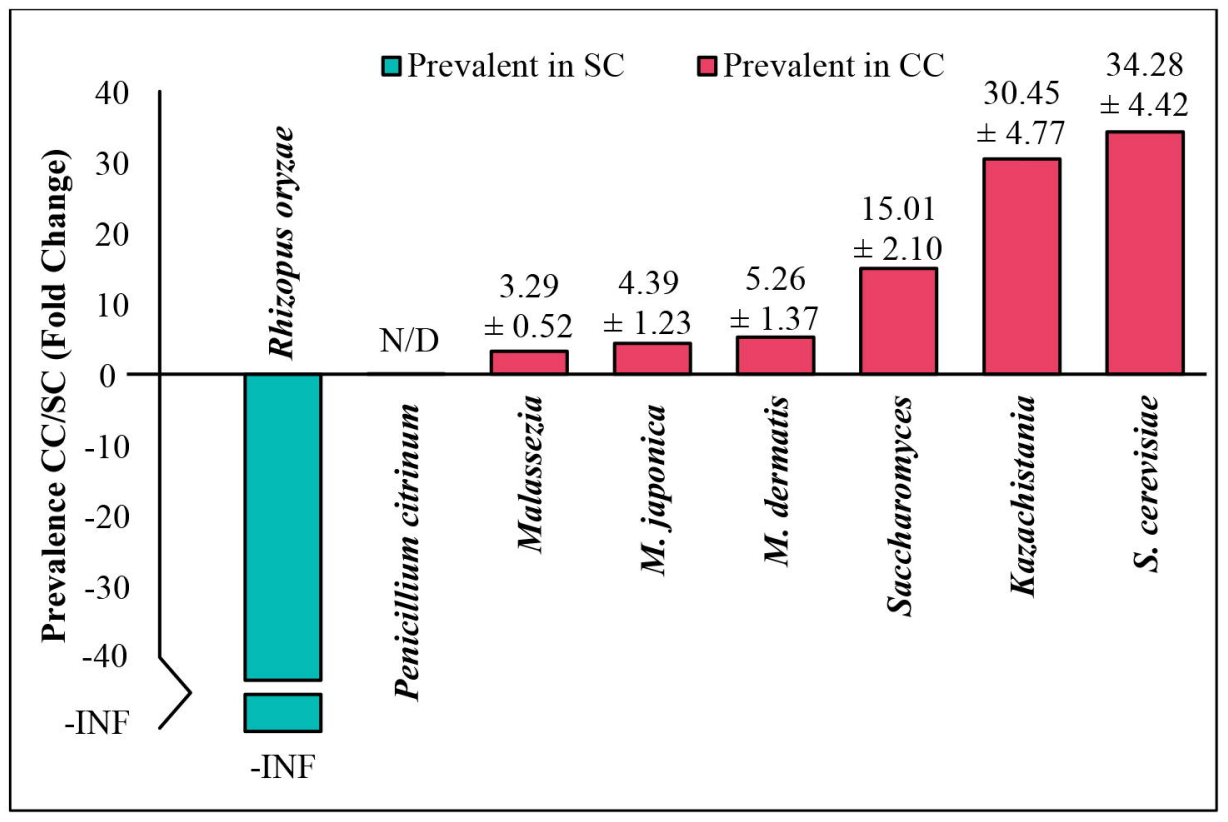

